\title{
Parámetros que Afectan la Corriente de Neutro en Presencia de Armónicos
}

\author{
Juan A. Suárez, Guillermo F. di Mauro, Daniel O. Anaut y Carlos Agüero \\ Universidad Nacional de Mar del Plata, Departamento de Ingeniería Eléctrica. Facultad de \\ Ingeniería, Juan B. Justo 4302, Mar del Plata, Pcia. Buenos Aires-Argentina \\ (e-mail: jsuarez@fi.mdp.edu.ar)
}

\section{Resumen}

Este trabajo analiza potenciales variables que afectan a la corriente de neutro cuando los receptores conectados presentan características no lineales. Se simularon en el programa Alternative Transients Program distintos escenarios combinando el tipo y número de unidades de cargas, alimentadas sucesivamente con tensiones sinusoidales, simétricas, balanceadas, asimétricas y desbalanceadas. También ha sido estudiado el efecto de armónicos en la tensión de alimentación. A los efectos de contrastar resultados se registraron mediciones de campo en un centro de cómputos. Como producto de las simulaciones con fuentes asimétricas y desbalanceadas se comprobó variaciones en la corriente de neutro de hasta un 5\%. Los análisis de los efectos diversidad y atenuación registraron variaciones más importantes: $10 \%$ y $20 \%$ respectivamente. Los resultados muestran que las asimetrías y desbalances en las fuentes de alimentación tienen una ligera incidencia en la corriente de neutro, mientras que los efectos diversidad y atenuación fueron más importantes.

\section{Parameters Affecting Neutral Currents in the Presence of Harmonics}

\begin{abstract}
This work analyzes potentials variables that affect neutral currents when the receptors connected present nonlinear characteristics. Different scenarios were simulated in the Alternative Transients Program combining the type and number of load units, successively fed with sinusoidal, symmetric, balanced, asymmetric and unbalanced power supply voltage. The effect of harmonics on source voltage was also studied. Field measurements were registered in a computer center to contrast results. A variation of up to $5 \%$ in the neutral current was registered as product of the simulations with asymmetric and unbalanced sources. The analysis of the diversity effect showed variations of a little more than $10 \%$ whereas the attenuation effect caused a $20 \%$ reduction. The results show that the asymmetries and unbalances in the feeding sources have a slight incidence on neutral currents, whereas the diversity and attenuation effects are more important.
\end{abstract}

Keywords: harmonics, neutral currents, simulation, Alternative Transients Program 


\section{INTRODUCCIÓN}

El uso cada vez más frecuente de cargas no lineales como computadoras personales (PCs), lámparas fluorescentes compactas (LFCs), fuentes conmutadas de diferentes equipos electrónicos, etc. ha motivado en los últimos años la atención de los investigadores de la ingeniería eléctrica en los distintos problemas provocados por la degradación de la calidad de la energía.

Las cargas no lineales demandan corrientes no sinusoidales y debido a la impedancia de cortocircuito de la red, provocan la aparición de armónicos de tensión en el punto de conexión (Ghinjselen et al., 2003). El incremento de los armónicos de baja frecuencia en las redes de distribución, que generalmente coinciden con los armónicos dominantes de cargas no lineales, ocasiona problemas para los receptores conectados al mismo punto, como fallos de operación en los equipos de medida, control y protección, además de un aumento de temperatura en conductores y generadores.

Otro aspecto importante a considerar cuando estas cargas son conectadas a sistemas trifásicos, es el nivel de corriente en el conductor de neutro que suele ser elevado y superar a la corriente de fase. Estudios realizados en Estados Unidos de América, revelaron que más del $20 \%$ de los complejos comerciales tienen en sus instalaciones corrientes de neutro superiores a las corrientes de línea (Jou et al., 2005). Contrariamente al pensamiento tradicional, esfuerzos hechos para balancear las cargas entre las fases pueden contribuir a incrementar la magnitud de la corriente de neutro. La excesiva corriente de neutro en presencia de cargas no lineales es un tema de interés en la investigación de la calidad de la energía ampliamente tratado por la literatura: Paraiso et al. (2005); Batrinu et al. (2007); Chan et al. (2007); Chicco et al. (2007); Demoulias et al. (2008); Larsson et al. (2008); Lowenstein (2008); Rodríguez et al. (2008); Song et al. (2008).

Sumando efectos no deseados, producto de la presencia de armónicos, se encuentra el calentamiento debido a pérdidas adicionales en máquinas y transformadores. En el caso de los transformadores existirán pérdidas suplementarias debido al efecto pelicular, histéresis y Foucault. En cables del sistema de alimentación es de esperar un aumento de pérdidas por efecto Joule, motivado por el incremento de la resistencia aparente del conductor con la frecuencia (Brugnoni, 2007).

En el mismo contexto, este trabajo propone analizar el comportamiento de la corriente de neutro de un sistema trifásico en función de la variación de parámetros inherentes tanto a la carga como a la red de alimentación. Para lograr este objetivo se simularon en el Alternative Transients Program (ATP), distintos modelos de cargas combinados con diferentes condiciones en las fuentes de alimentación. Complementariamente con ello, se realizaron mediciones de campo en un centro de cómputos para contrastar el comportamiento de la corriente de neutro con el obtenido por simulación.

\section{Revisión Teórica}

Se presenta en esta sección una revisión de conceptos asociados al estudio de las corrientes provocadas por cargas no lineales.

En un sistema eléctrico trifásico, la corriente de neutro es el vector suma de las tres corrientes de líneas. Si el sistema de alimentación es balanceado, con una simetría de sus ondas en $120^{\circ}$ eléctricos y con carga lineal trifásica perfectamente balanceada, la corriente de neutro es igual a cero. En la práctica se espera que en un sistema trifásico de cuatro hilos, medianamente balanceado, la corriente que circula por el neutro sea apenas un $20 \%$ de la corriente de fase. Muy distinto es el panorama si las cargas no son lineales. La aparición de los componentes armónicos impares, en particular los múltiplos de tres, inciden en el aumento de la corriente de neutro aunque el sistema se encuentre balanceado.

En un sistema trifásico de cuatro hilos con cargas no lineales balanceadas, la corriente en el neutro dependerá básicamente del contenido armónico de las corrientes de fases. 
Si se suponen cargas no lineales con componentes armónicos impares, se puede descomponer a cada una de las corrientes de fase usando la transformada de Fourier:

$$
\begin{aligned}
& I_{R}(t)=I_{1} \operatorname{sen}\left(\omega t+\alpha_{1}\right)+I_{3} \operatorname{sen}\left(3 \omega t+\alpha_{3}\right)+I_{5} \operatorname{sen}\left(5 \omega t+\alpha_{5}\right)+\ldots \\
& I_{S}(t)=I_{1} \operatorname{sen}\left(\omega t-\frac{2 \pi}{3}+\alpha_{1}\right)+I_{3} \operatorname{sen}\left(3\left(\omega t-\frac{2 \pi}{3}\right)+\alpha_{3}\right)+I_{5} \operatorname{sen}\left(5\left(\omega t-\frac{2 \pi}{3}\right)+\alpha_{5}\right)+\ldots \\
& I_{T}(t)=I_{1} \operatorname{sen}\left(\omega t-\frac{4 \pi}{3}+\alpha_{1}\right)+I_{3} \operatorname{sen}\left(3\left(\omega t-\frac{4 \pi}{3}\right)+\alpha_{3}\right)+I_{5} \operatorname{sen}\left(5\left(\omega t-\frac{4 \pi}{3}\right)+\alpha_{5}\right)+\ldots
\end{aligned}
$$

La expresión de la corriente en el neutro en un sistema trifásico simétrico y balanceado puede ser escrita como la sumatoria de las corrientes de las fases.

$$
I_{n(t)}=I_{R(t)}+I_{S(t)}+I_{T(t)}=\sum 3 I_{6 k+3} \operatorname{sen}\left((6 k+3) \omega t+a_{6 k+3}\right)
$$

La ecuación (4), muestra que la corriente en el neutro bajo condiciones balanceadas, es producida por las componentes de secuencia cero $(3,9,15,21 \ldots)$ de las corrientes de las fases.

Se define con $\rho_{1}$ a la relación:

$$
\rho_{1}=\frac{I_{N}}{I_{f}}=\frac{\sqrt{\sum\left(3 I_{6 k+3}\right)^{2}}}{\sqrt{\sum\left(3 I_{6 k+1}\right)^{2}+\sum\left(3 I_{6 k+3}\right)^{2}+\sum\left(3 I_{6 k+5}\right)^{2}}}
$$

En un circuito simétrico y balanceado, la relación $\rho_{1}$ se incrementa con el aumento del armónico de tercer orden y con la disminución del primero y quinto de la corriente de fase.

Si las corrientes de fases solo tienen componentes armónicos impares $I_{2 n+1}$ con $I_{2 n+1}=q^{n} I_{1}\left(I_{3}=q \cdot I_{1}\right.$, $\left.I_{5}=q^{2} . I_{1}, I_{7}=q^{3} . I_{1} \ldots\right)$, Desmet et al. (2001), deducen que la relación entre la corriente de neutro y fase resulta ser:

$$
\rho_{1}=3 \frac{q}{\sqrt{1+q^{2}+q^{4}}}
$$

Se demuestra que la corriente de neutro es máxima e igual a $\sqrt{3}$ veces la corriente de línea cuando $q=1$, condición que se cumple cuando todos los componentes armónicos tienen el mismo peso.

Arthur y Shanahan (1996), expresan la ecuación de $\rho_{1}$ en función del índice de distorsión armónica total de corriente $\left(\mathrm{THD}_{\mathrm{l}}\right)$ como sigue:

$$
\rho_{2}=3 \frac{\mathrm{THD}_{1}}{\sqrt{1+\mathrm{THD}_{\mathrm{I}}^{2}}}
$$

La Ecuación 7 es válida bajo el supuesto que los armónicos son impares y el de tercer orden es el más preponderante, alcanzando el máximo $(1,73)$ cuando el índice de distorsión de corriente es del $70,7 \%$.

A partir de un sistema desbalanceado, con dos fases (R y S) cargadas al $100 \%$ y la tercera (T) con carga variable desde 0 hasta 100\%, Cadavid y Gallego (2003), reescriben la Ecuación 7 como:

$$
\rho_{3}=\sqrt{(1-m)^{2}+(6 m+3) \frac{T H D_{1}^{2}}{100^{2}+T_{1}^{2}}}
$$


Los diagramas de las Figuras 1 y 2 representan la variación de $\rho_{1}$ y $\rho_{3}$ en función de "q" y "m" respectivamente.

\section{METODOLOGÍA}

En este apartado se describen las metodologías empleadas en distintos escenarios en los cuales se variaron las condiciones de carga y alimentación de una red trifásica con conductor de neutro.

El primer paso consistió en ensayar el comportamiento de dos tipos de cargas: una con alta distorsión de corriente (THD, del 90\%), combinación de computadora y monitor ( $\mathrm{PC}$ ) y una segunda con un $\mathrm{THD}_{1}$ relativamente bajo $(30 \%)$ representada por una lámpara fluorescente (LF), Suárez et al. (2005). De estos ensayos se obtuvieron las amplitudes y ángulos de fase de los componentes armónicos. Los espectros de frecuencias de ambas cargas se resumen en la Figura 3.

Con los datos obtenidos, se modelaron en el ATPDraw (preprocesador gráfico del ATP) los circuitos eléctricos de la fuente trifásica de alimentación y de las cargas seleccionadas (PC y LF). Uno de los sistemas de alimentación utilizado, es una red trifásica con neutro, cuyo esquema equivalente es el de la Figura 4, (Sistema 1), empleando fuentes "tipo 14" del ATP, suficientes para el análisis de simetría y desbalance en las tensiones. La impedancia equivalente de la combinación transformadorlínea, $Z_{e q}=R_{e q}+j X_{e q}$, es igual a $0.5 \Omega+j 0.09 \Omega$. La impedancia del neutro $Z_{N}=R_{N}+j X_{N}$, es igual a

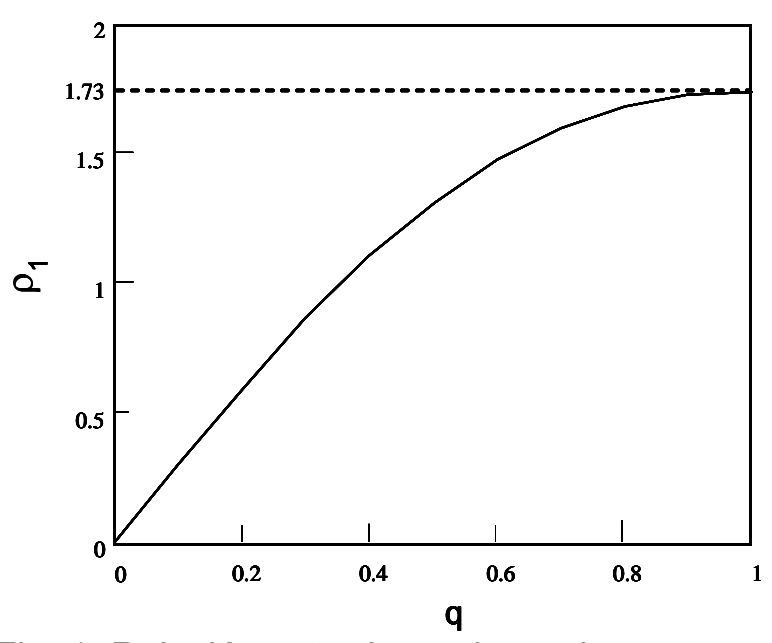

Fig. 1: Relación entre la corriente de neutro y de fase en función de "q"

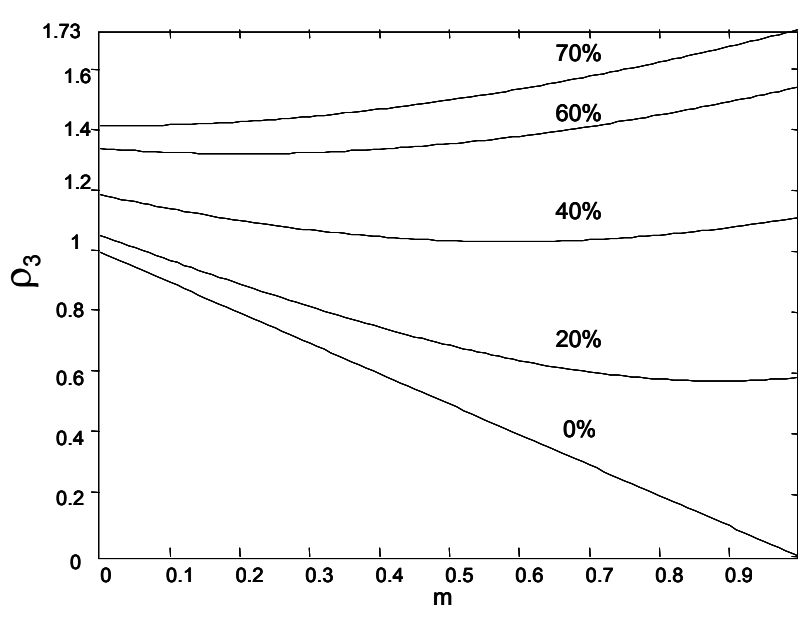

Fig. 2: Relación entre la corriente de neutro y de fase en función de "m"

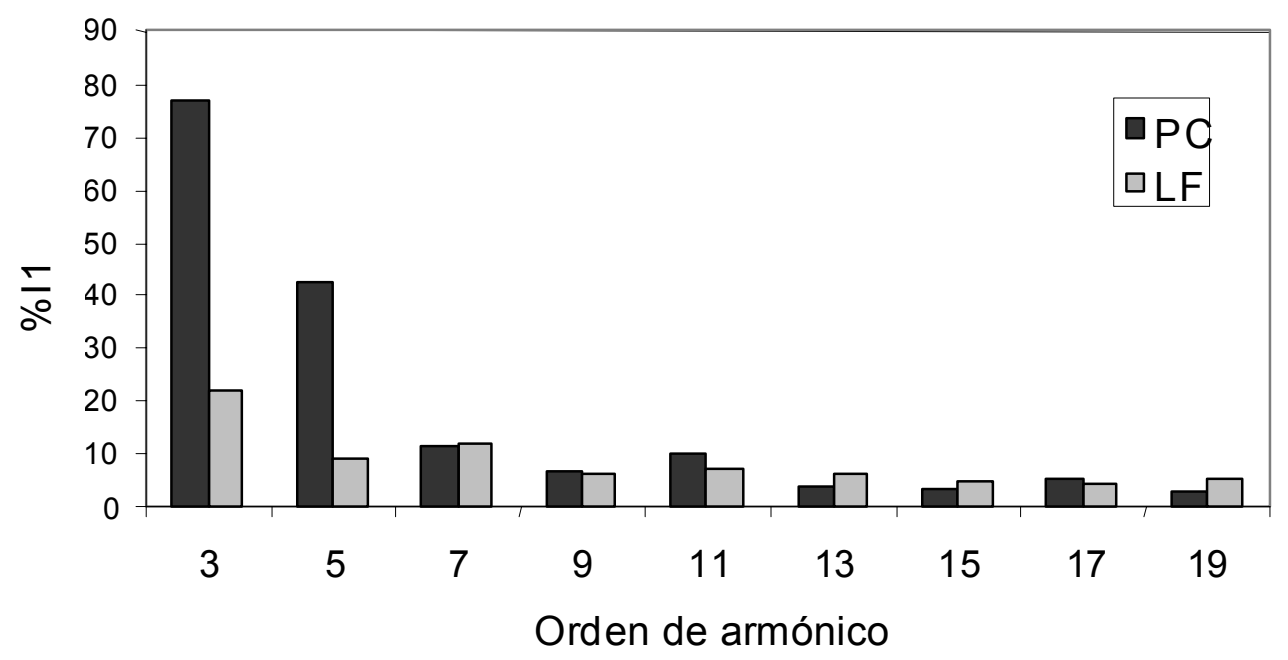

Fig. 3: Espectro de frecuencias de corrientes de PC y LF 
$0.1 \Omega+j 0.02 \Omega$. Para analizar la influencia de armónicos en la tensión, el esquema a simular es el de la Figura 5, donde las fuentes han sido reemplazadas por las del tipo HFS, Harmonic Frecuency Scan (Sistema 2). La tensión de cada una de las fases se ajustó a 220 V eficaz.

\section{SIMULACIÓN Y RESULTADOS}

Las simulaciones en el ATP se realizan con distintas condiciones de cargas para regímenes balanceados y desbalanceados.

\section{Alimentación balanceada y simétrica con cargas balanceadas}

Para analizar el comportamiento de la corriente de neutro ante distintos tipos de cargas no lineales, a cada una de las fases de la red trifásica del Sistema 1, se las cargó sucesivamente con una PC, y luego con una LF. En cada una de las simulaciones se registraron las corrientes de $I_{f}$ y $I_{N}$ con sus respectivos espectros armónicos y se calcularon las relaciones $I_{N} / I_{f}$ definidas en Ecuación 6 y 7 junto a la simulada $\left(\rho_{\mathrm{s}}\right)$. Los valores obtenidos se resumen en la Tabla 1.

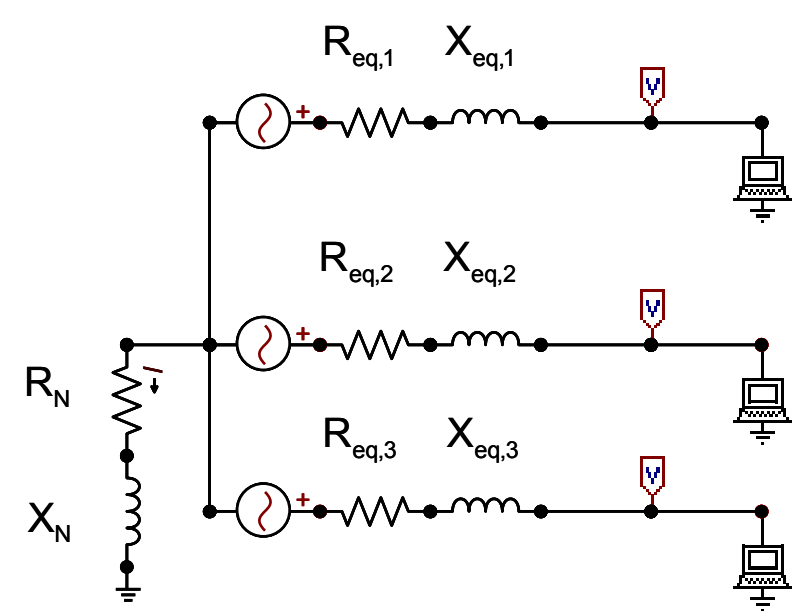

Fig. 4: Sistema 1, utilizando fuentes tipo 14 del ATP

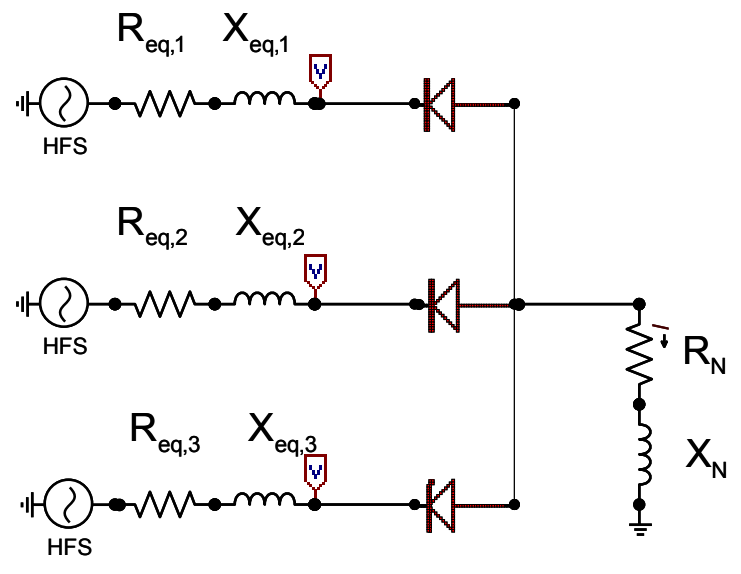

Fig. 5: Sistema 2, utilizado fuentes HFS

Tabla 1: Valores $\rho_{1}, \rho_{2}, \rho_{\mathrm{s}}$ para las cargas simuladas

\begin{tabular}{|c|c|r|r|r|r|}
\hline Carga & $\begin{array}{c}\mathrm{THD}_{1} \\
{[\%]}\end{array}$ & $\mathrm{q}=\mathrm{I}_{3} / /_{1}$ & $\rho_{1}$ & $\rho_{2}$ & $\rho_{\mathrm{s}}$ \\
\hline $1 \times \mathrm{LF}$ & 30.49 & 0.221 & 0.647 & 0.875 & 0.679 \\
\hline $1 \times \mathrm{PC}$ & 90.04 & 0.770 & 1.657 & 1.732 & 1.726 \\
\hline
\end{tabular}

De la Tabla 1 se deduce:

- Para la carga PC, $\rho_{2}$ se aproxima al valor simulado, puesto que el $90 \%$ del $T H D_{1}$ está provocado por una alta componente de tercer orden, condición necesaria para que la Ecuación 7 sea válida. Más del $85 \%$ del valor eficaz de la suma de todos los componentes armónicos pertenece al de orden 3.

- En el otro extremo para la LF, encontramos $\rho_{1}$ más próximo a $\rho_{\mathrm{s}}$, puesto que en este caso el armónico 3 no es tan preponderante en la composición del $\mathrm{THD}_{\text {। }}$ con tan solo un $22 \%$ de la componente fundamental.

\section{Red balanceada y simétrica con cargas desbalanceadas}

A la misma configuración de la red trifásica del Sistema 1, se alimentó las fases $\mathrm{R}$ y $\mathrm{S}$ con diez unidades idénticas, primero con PCs y luego con LFs. La carga en la fase T se incrementó de a dos unidades, desde cero hasta 10. 
Del resultado de las simulaciones se grafican en la Figura $6, I_{N} / I_{f}$ en función del porcentaje de carga en la fase $\mathrm{T}$. Como se observa los valores de $\rho_{\mathrm{s}}$ pertenecientes a la cargas con distorsión de corriente superior al $70 \%$ (PCs) aumentan con el incremento del porcentaje de la carga $\mathrm{T}$ y su curva se aproxima a los máximos obtenidos con la Ecuación 8.

Por el contrario, para las LFs los valores de corriente de neutro, sensiblemente inferiores a los máximos teóricos, disminuyen en la medida que el sistema se equilibra.

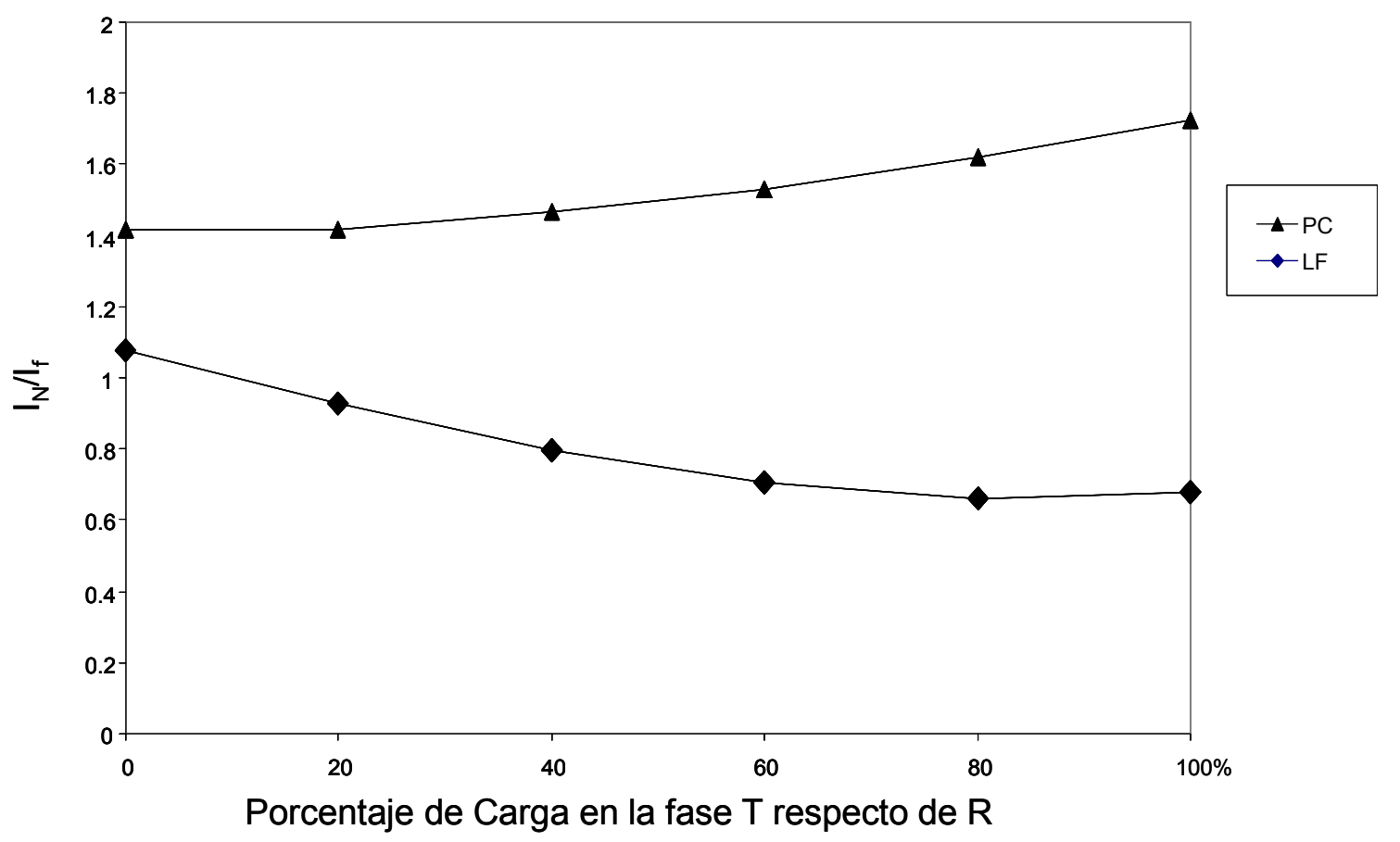

Fig. 6: Relación $I_{N} / I_{f}$ en función de la variación porcentual de carga en la fase $T$

\section{Red de alimentación desbalanceada}

Para estudiar la influencia en la corriente de neutro que provoca el desbalance en las tensiones de fases y la asimetría entre sus fasores se utilizó como carga, para cada una de las fases del Sistema 1 , un modelo de rectificador monofásico del tipo puente, con una potencia de $300 \mathrm{~W}$ y un $T H D_{1}$ del $87 \%$, (Figura 7). El mismo representa la corriente de consumo de muchos tipos de cargas no lineales.

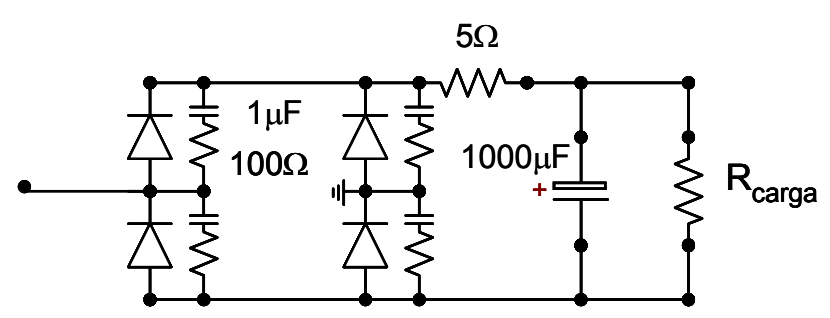

Fig. 7: Modelo rectificador tipo puente simulado en el ATP

Cuatro simulaciones fueron realizadas para analizar el impacto del desbalance. Se varió el valor eficaz de cada una de las tensiones de fases desde 0 hasta $\pm 10 \%$ de la referencia (220 V). Los datos de la Tabla 2 permiten demostrar que prácticamente las diferencias relativas porcentuales en la corriente de neutro $\left(\Delta_{r} \%\right)$ respecto al valor con tensión balanceada, apenas alcanzan el $-1 \%$. Esto es debido a que una pequeña disminución del tercer armónico en la corriente del neutro $\left(I_{3, N}\right)$ se compensa con un ligero aumento de la componente de primer orden $\left(I_{1, N}\right)$. 
Red de alimentación asimétrica

Utilizando la configuración anterior, ahora manteniendo el sistema balanceado en $220 \mathrm{~V}$, realizamos simulaciones modificando la asimetría de las fuentes, con variaciones de ángulos entre $\pm 2.5 \%$ a $\pm 10 \%$.

En la Tabla 3 se resumen los valores de variación porcentual de la corriente de neutro en función de la asimetría de la tensión de alimentación. En la misma observamos disminuciones poco significativas en $I_{N}$ y justificaciones muy similares a las del ensayo anterior: leves disminuciones del tercer armónico en la corriente de neutro se contrarrestan con pequeños aumentos de $\mathrm{I}_{1, \mathrm{~N}}$.

Tabla 2: Variación porcentual de la corriente de neutro en función del desbalance de la tensión de alimentación.

\begin{tabular}{|c|c|c|c|c|c|c|c|}
\hline $\begin{array}{c}\text { Variación } \\
\text { de Amplitud } \\
\text { de Tensión }\end{array}$ & $\begin{array}{c}\mathrm{U}_{\mathrm{R}} \\
{[\mathrm{V}]}\end{array}$ & $\begin{array}{c}\mathrm{U}_{\mathrm{S}} \\
{[\mathrm{V}]}\end{array}$ & $\begin{array}{c}\mathrm{U}_{\mathrm{T}} \\
{[\mathrm{V}]}\end{array}$ & $\begin{array}{c}\mathrm{I}_{\mathrm{N}} \\
{[\mathrm{A}]}\end{array}$ & $\begin{array}{c}\mathrm{I}_{1, \mathrm{~N}} \\
{[\mathrm{~A}]}\end{array}$ & $\begin{array}{l}\mathrm{I}_{3, \mathrm{~N}} \\
{[\mathrm{~A}]}\end{array}$ & $\Delta_{\mathrm{r}} \%$ \\
\hline $\pm 0 \%$ & 220 & 220 & 220 & 2.341 & 0.0005 & 2.3407 & 0 \\
\hline $\pm 2.5 \%$ & 226 & 215 & 220 & 2.339 & 0.0446 & 2.3264 & -0.61 \\
\hline $\pm 5 \%$ & 209 & 231 & 220 & 2.34 & 0.0934 & 2.326 & 0.63 \\
\hline $\pm 10 \%$ & 242 & 198 & 220 & 2.337 & 0.1877 & 2.3164 & -1.04 \\
\hline
\end{tabular}

Tabla 3: Variación porcentual de la corriente de neutro en función de la asimetría de la tensión de alimentación.

\begin{tabular}{|c|c|c|c|c|c|c|c|}
\hline $\begin{array}{c}\text { Variación } \\
\text { de Ángulo } \\
\text { de la } \\
\text { tensión }\end{array}$ & $\begin{array}{c}\varphi_{\mathrm{R}} \\
\left.{ }^{\circ}\right]\end{array}$ & $\begin{array}{c}\varphi_{\mathrm{S}} \\
\left.{ }^{\circ}\right]\end{array}$ & $\begin{array}{c}\varphi_{\mathrm{T}} \\
\left.{ }^{\circ}\right]\end{array}$ & $\begin{array}{c}\mathrm{I}_{\mathrm{N}} \\
{[\mathrm{A}]}\end{array}$ & $\begin{array}{c}\mathrm{I}_{1, \mathrm{~N}} \\
{[\mathrm{~A}]}\end{array}$ & $\begin{array}{c}\mathrm{I}_{3, \mathrm{~N}} \\
{[\mathrm{~A}]}\end{array}$ & $\Delta_{\mathrm{r}} \%$ \\
\hline $0 \%$ & 0 & 120 & 240 & 2.341 & 0.001 & 2.341 & 0.00 \\
\hline $\pm 2.5 \%$ & 0 & 123 & 243 & 2.337 & 0.055 & 2.322 & -0.17 \\
\hline $\pm 5 \%$ & 0 & 126 & 246 & 2.326 & 0.110 & 2.301 & -0.64 \\
\hline $\pm 10 \%$ & 0 & 132 & 252 & 2.283 & 0.219 & 2.222 & -2.49 \\
\hline
\end{tabular}

\section{Red con armónicos de tensión}

Para investigar los efectos que producen los componentes armónicos de tensión de la fuente trifásica de alimentación en la corriente $I_{N}$, se utilizó el Sistema 2 . A cada una de las tensiones de fase se le incorporó un armónico de tercer orden de $10 \mathrm{~V}$ de valor eficaz y con un ángulo de fase inicial $\beta_{3}=0^{\circ}$, respecto al componente fundamental de la tensión.

Usando como carga el mismo modelo de rectificador de $300 \mathrm{~W}$ se realizaron tres simulaciones distintas variando el ángulo de fase $\beta_{3}$ en $0^{\circ}, 90^{\circ}$ y $180^{\circ}$.

Los resultados son resumidos en la Tabla 4. Las mayores fluctuaciones se registran para $\beta_{3}=0^{\circ}$ y $\beta_{3}=180^{\circ}$, provocadas por aumento y disminución del tercer armónico de corriente en correspondencia con la mayor y menor amplitud de la onda de tensión respectivamente.

En la Figura 8 se muestran los oscilogramas de corrientes, el de $I_{N}$ con tensión sinusoidal, junto con los $I_{N}$ obtenidos en las simulaciones con el ángulo de fase del tercer armónico en $\beta_{3}=0^{\circ}$ y $\beta_{3}=180^{\circ}$. 


\section{Análisis del efecto de diversidad}

Para el analizar el impacto que el efecto diversidad provoca en la corriente de neutro se realizaron simulaciones en dos escenarios distintos:

\section{Escenario 1:}

Para esta evaluación se conectaron seis modelos idénticos del rectificador puente con una potencia individual de $265 \mathrm{~W}$, a cada una de las fases del Sistema 1, teniendo como objetivo analizar el comportamiento de la corriente de neutro. A los efectos de no superponer el efecto atenuación, fue despreciada la impedancia $Z_{\text {eq. }}$.

\section{Escenario 2:}

A cada rectificador se le varió el nivel de potencia en valores de $\pm 35 \%$, a partir de un valor medio de $265 \mathrm{~W}$, modificando la resistencia de carga ( $\left.R_{\text {carga }}\right)$. Los oscilogramas individuales de las corrientes de consumo de cada carga obtenidos en la simulación, tienen una apariencia similar a los de la Figura 7. Las variaciones en el nivel de potencia de cada uno de los convertidores provocan diferencias en los ángulos de fase de los componentes armónicos.

Los datos finales de las simulaciones en los dos escenarios, comparando las corrientes de fase y neutro se resumen en la Tabla 5 . El factor diversidad provoca en la corriente de neutro y para las condiciones analizadas, una reducción de $11.1 \mathrm{~A}$ a $9.7 \mathrm{~A}$.

Tabla 4: Corriente de neutro y sus componentes armónicos en función de la variación del ángulo de fase del armónico de tensión.

\begin{tabular}{|c|c|c|c|c|c|c|c|c|}
\hline \multicolumn{2}{|c|}{$\begin{array}{c}\text { Componente } \\
\text { Armónico Tensión }\end{array}$} & \multirow{2}{*}{$\begin{array}{c}\mathrm{THD}_{\mathrm{u}} \\
{[\%]}\end{array}$} & \multirow{2}{*}{$\begin{array}{l}\text { THD } \\
\text { [\%] }\end{array}$} & \multirow{2}{*}{$\begin{array}{l}I_{N} \\
{[A]}\end{array}$} & \multirow{2}{*}{$\begin{array}{l}I_{3, \mathrm{~N}} \\
{[\mathrm{~A}]}\end{array}$} & \multirow{2}{*}{$\begin{array}{l}l_{9, N} \\
{[A]}\end{array}$} & \multirow{2}{*}{$\begin{array}{l}\mathrm{I}_{15, \mathrm{~N}} \\
{[\mathrm{~A}]}\end{array}$} & \multirow{2}{*}{$\Delta_{\mathrm{r}} \%$} \\
\hline $\begin{array}{l}\beta_{3} \\
{\left[{ }^{\circ}\right]}\end{array}$ & $\begin{array}{c}\mathrm{U}_{3, \mathrm{R}} \\
{[\mathrm{V}]} \\
\end{array}$ & & & & & & & \\
\hline 0 & 0 & 0 & 87 & 2.34 & 2.34 & 0.21 & 0.09 & 0.00 \\
\hline 0 & 10 & 4.53 & 95 & 2.55 & 2.54 & 0.07 & 0.02 & 8.76 \\
\hline 90 & 10 & 4.53 & 89 & 2.38 & 2.37 & 0.18 & 0.08 & 1.77 \\
\hline 180 & 10 & 4.53 & 76 & 2.09 & 2.07 & 0.29 & 0.10 & -10.61 \\
\hline
\end{tabular}

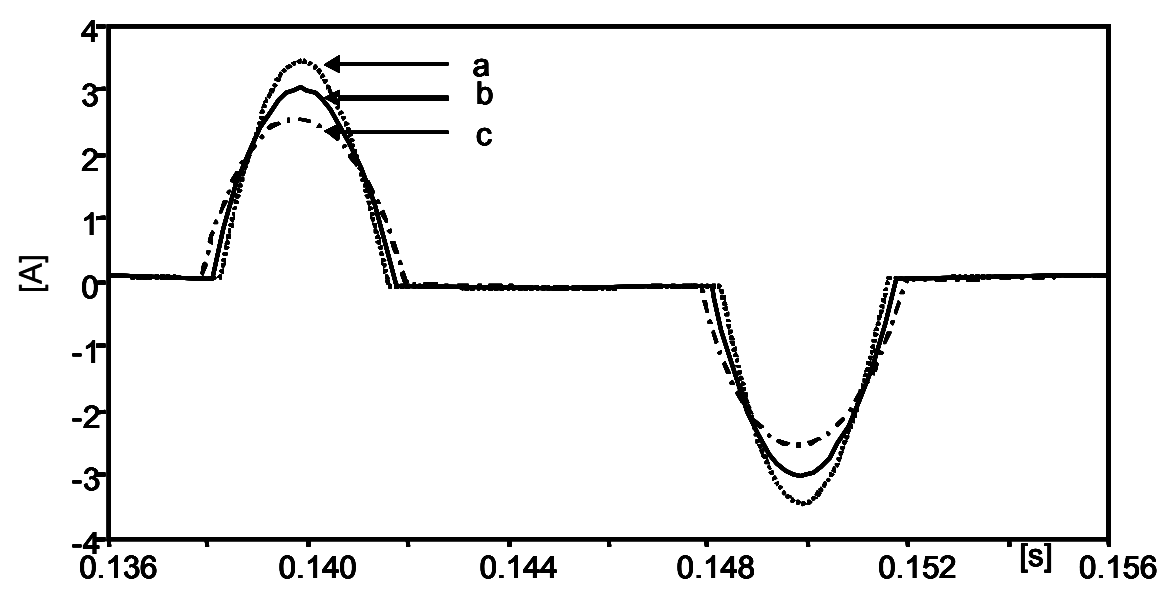

Fig. 8: Corriente de Neutro, con tensión (b) sinusoidal, (a) 3er armónico $\beta_{3}=0^{\circ}$, (c) 3er armónico $\beta_{3}=180^{\circ}$ 
Tabla 5: Valores eficaces de corrientes en fase y neutro para cargas idénticas y distintas

\begin{tabular}{|l|c|c|c|c|c|c|c|c|c|c|}
\hline $\begin{array}{c}\text { Tipo } \\
\text { Simulación }\end{array}$ & $\begin{array}{c}I_{f} \\
{[A]}\end{array}$ & $\begin{array}{c}I_{N} \\
{[A]}\end{array}$ & $\begin{array}{c}I_{3, f} \\
{[A]}\end{array}$ & $\begin{array}{c}I_{3, N} \\
{[A]}\end{array}$ & $\begin{array}{c}I_{9, f} \\
{[A]}\end{array}$ & $\begin{array}{c}I_{9, N} \\
{[A]}\end{array}$ & $\begin{array}{c}I_{15, f} \\
{[A]}\end{array}$ & $\begin{array}{c}I_{15, N} \\
{[A]}\end{array}$ & $\begin{array}{c}I_{21, f} \\
{[A]}\end{array}$ & $\begin{array}{c}I_{21, N} \\
{[A]}\end{array}$ \\
\hline $\begin{array}{l}\text { Cargas } \\
\text { Iguales }\end{array}$ & 7.58 & 11.13 & 3.703 & 11.1 & 0.194 & 0,584 & 0,119 & 0,358 & 0,029 & 0,088 \\
\hline $\begin{array}{l}\text { Cargas } \\
\text { Distintas }\end{array}$ & 7.23 & 9.67 & 3.222 & 9.7 & 0,065 & $0,190,083$ & 0,247 & 0,004 & 0,009 \\
\hline
\end{tabular}

\section{Análisis del efecto atenuación}

Para analizar la influencia de la impedancia de línea de alimentación en la corriente de neutro, nueve modelos de rectificadores con una potencia individual media de $420 \mathrm{~W}$ son conectados a cada una de las fases del Sistema 1.

Como referencia corremos una primera simulación sin conectar impedancia alguna en la línea. Posteriormente manteniendo el valor de $R_{\text {eq }}$ constante e igual a $0.5 \Omega$, variamos la $X_{\text {eq }}$, obteniendo simulaciones con una relación de $\mathrm{X}_{\mathrm{eq}} / \mathrm{R}_{\text {eq }}$ desde 0 hasta 2.5 .

En la Tabla 6 se resumen para cada una de las relaciones $X_{\text {eq }} / R_{\text {eq }}$ los valores de corrientes de neutro obtenidos. Un aumento de la relación $X_{\text {eq }} / R_{\text {eq }}$ produce una mayor deformación en la onda de tensión, que provoca una reducción del tercer armónico y por ende una menor corriente en el neutro.

En la Fig. 9, se muestra el espectro de frecuencias de la corriente en el neutro, para las condiciones analizadas.

\section{Medición en un centro de cómputos}

Con el propósito de cotejar las simulaciones propuestas, mediciones en el tablero principal de un centro de cómputos de una empresa prestadora de servicios públicos fueron realizadas.

Los registros de distorsión de corriente grabados con un analizador de redes con lecturas cada 5 minutos, muestran la evolución de los índices de distorsión de corrientes de líneas a lo largo del día (Fig. 10). Entre las 15 y 17 horas, cuando una parte importante de las PCs son apagadas, se observa

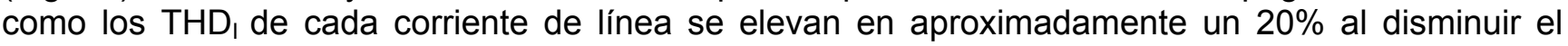
efecto conjunto de diversidad y atenuación.

En la Fig. 11 se grafica la evolución de los componentes armónicos de la corriente de neutro para el mismo período. Como es de esperar por el tipo de carga, se destaca la fuerte presencia del armónico tercero.

El valor máximo de la corriente de neutro se mantuvo en aproximadamente $27 \mathrm{~A}$ en los momentos de mayor actividad, mientras las corrientes de líneas máximas registradas fueron de $29.5 \mathrm{~A}, 18.8 \mathrm{~A}$ y 16.5 A.

Tabla 6: Comportamiento de la corriente de neutro por variación de la impedancia de línea

\begin{tabular}{|c|c|c|c|c|c|}
\hline $\begin{array}{l}\mathrm{R}_{\mathrm{eq}} \\
{[\Omega]}\end{array}$ & $\begin{array}{l}\mathrm{X}_{\mathrm{eq}} \\
{[\Omega]}\end{array}$ & $\mathrm{X}_{\mathrm{eq}} / \mathrm{R}_{\mathrm{eq}}$ & $\begin{array}{c}\mathrm{I}_{\mathrm{N}} \\
{[\mathrm{A}]}\end{array}$ & $\begin{array}{c}\mathrm{THD}_{1} \\
{[\%]}\end{array}$ & $\Delta_{\mathrm{r}} \%$ \\
\hline 0.5 & 0 & 0 & 32.95 & 105 & 0 \\
\hline 0.5 & 0.63 & 1.26 & 30.66 & 82 & -7.47 \\
\hline 0.5 & 1.26 & 2.5 & 25.94 & 71 & -21.27 \\
\hline
\end{tabular}




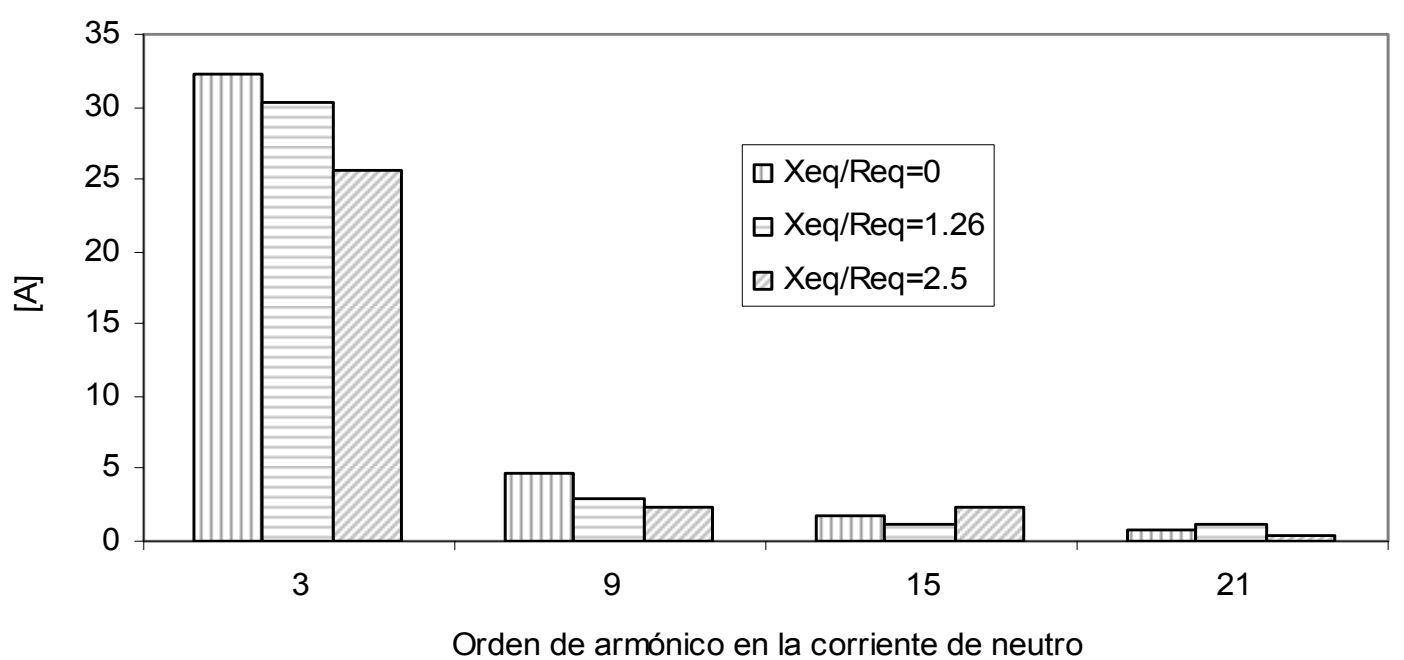

Fig. 9: Espectro frecuencias en la corriente de neutro para distintas relaciones $X_{\text {eq }} / R_{\text {eq }}$

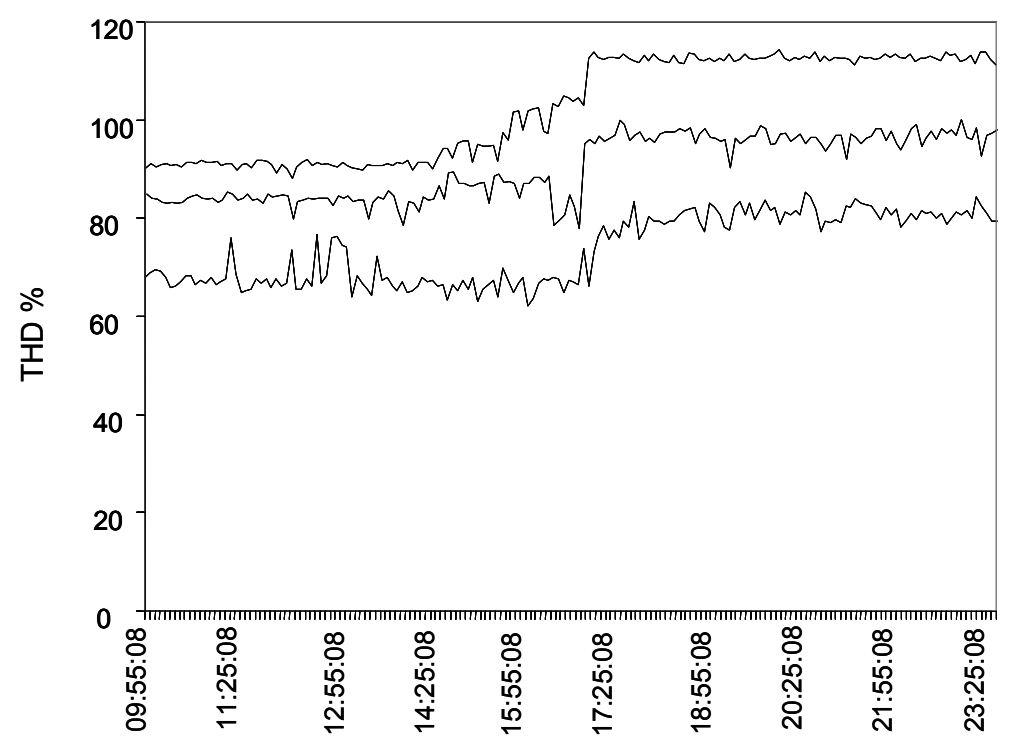

Fig. 10: Variación del THD, de las corrientes de líneas en un centro de cómputos.

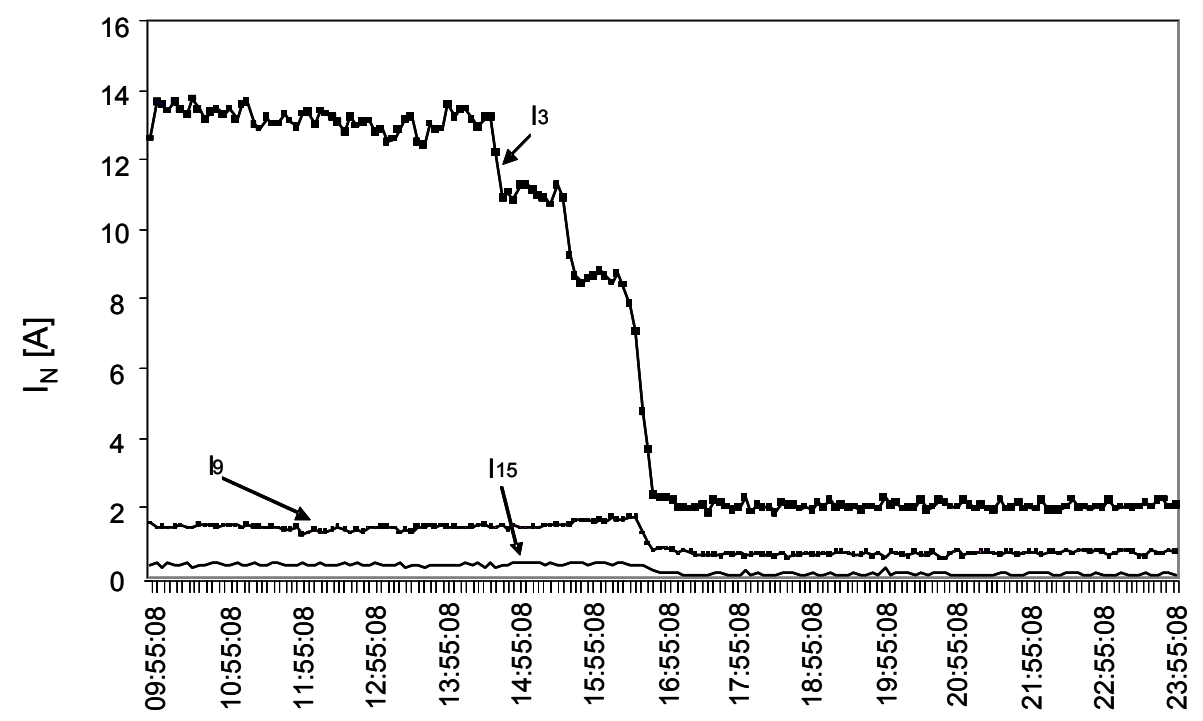

Fig. 11: Evolución de los componentes armónicos 3, 9 y 15 de la corriente de neutro registrados en un centro de cómputos 
De los valores registrados a lo largo del día en ningún caso la corriente de neutro superó a la máxima corriente medida en las líneas. De acuerdo a lo analizado en las simulaciones, cargas no lineales desbalanceadas, cuyos consumos tienen fuertes contenidos del tercer armónico, la corriente de neutro será menor que en régimen balanceado.

\section{DISCUSIÓN}

A partir de las simulaciones con modelos de PCs en el esquema del Sistema 1 y con las mediciones registradas en un centro de cómputos, se manifiesta que cuando una red trifásica alimenta cargas no lineales con un fuerte contenido del tercer armónico, la corriente en el neutro se aparta del máximo teórico (1.73 veces la corriente de línea), disminuyendo cuanto más desbalanceado es el sistema de cargas. En el sistema trifásico analizado en este trabajo, con dos fases cargadas al 100\% (10 PCs) y una tercera al $60 \%$ arrojó reducciones en la corriente de neutro del $12 \%$.

La influencia de una red con tensiones desbalanceadas o asimétricas tiene poca incidencia en la corriente de neutro, registrando ligeras fluctuaciones entre $1 \%$ y $2.5 \%$, conclusiones coincidentes a las obtenidas por Sainz et al. (2006) con modelos matemáticos de rectificadores. Ensayos con lámparas fluorescente compactas y con fuentes de tensión en condiciones similares a la del presente trabajo, realizadas por Desmet et al. (2001), arrojaron disminuciones entre el $1 \%$ y $5 \%$.

El impacto de una tensión distorsionada ha sido analizado, inyectando un componente de tercer orden a la red, con una amplitud de $14 \mathrm{~V}$ y con distintos ángulos de fase $\left(0^{\circ}, 90^{\circ}\right.$ y $\left.180^{\circ}\right)$. Para un defasaje de $0^{\circ}$ la corriente del neutro aumenta un $9 \%$ y disminuye un poco más del $10 \%$ para un ángulo de $180^{\circ}$.

Estudios realizados con PCs, por Paraiso et al. (2005) utilizando como herramienta computacional el PSPICE y con armónicos de quinto orden en la tensión, amplitud del $5 \%$ de la fundamental, registraron valores de un $10 \%$ de aumento con ángulo de $0^{\circ}$ y una disminución del $15 \%$ para un ángulo de $180^{\circ}$.

Finalmente los análisis de los efectos diversidad y atenuación reflejan siempre disminuciones en la corriente de neutro que depende y en mucho de la cantidad de unidades similares de cargas no lineales conectadas. Por un lado el efecto diversidad conectando 6 PCs, con ligeras variaciones en su nivel de potencia, impactaron en más de un $10 \%$ en la disminución de la corriente en el neutro, registrando las mayores variaciones en armónicos de orden 9,15 y 21.

La influencia del efecto atenuación, analizadas con nueve rectificadores por fase registraron reducciones del $20 \%$ en la corriente de neutro. Moore y Portugués (2003), estudiaron el comportamiento de 124 unidades de PCs, conectados sobre una misma fase, observando reducciones de hasta un $50 \%$ en el tercer armónico de la corriente de línea.

Este trabajo analizó además las variaciones en los armónicos para distintos regímenes de trabajo de PCs, en reposo, en procesamiento numérico, con acceso al disco duro, observando disminuciones en el tercer armónico de línea del $51 \%, 42 \%$ y $52 \%$ respectivamente, lo que supone una incidencia interesante al considerar el efecto diversidad.

\section{CONCLUSIONES}

Con simulaciones realizadas en el programa ATP, se han analizado los diferentes factores que pueden impactar en la corriente de neutro.

Un desbalance en las cargas puede provocar un aumento o disminución de la corriente en el conductor de neutro dependiendo de la relación $I_{3} / l_{1}$. Desbalances de $10 \%$ o asimetrías de hasta $10^{\circ}$ en la fuente de alimentación tienen un efecto muy leve sobre la corriente de neutro.

Componentes armónicos en la tensión de alimentación afecta el valor eficaz de la corriente de neutro, tanto más cuanto mayor sea el ángulo de fase del armónico. 
Los efectos diversidad y atenuación influyen notoriamente en la disminución de la corriente de neutro, cuando un número importante de cargas del mismo tipo se conectan a las fases de un sistema trifásico. Esto fue evidenciado con las mediciones realizadas en un centro de cómputos con un total de 80 PCs conectadas a un sistema de alimentación trifásico.

Tanto las simulaciones como las mediciones de campo demuestran que si bien en presencia de armónicos el nivel de la corriente de neutro puede ser importante, la suma de las causas estudiadas logra un significativo efecto de mitigación.

\section{NOMENCLATURA}

$\begin{array}{ll}I_{R(t)}, I_{S(t)}, I_{T(t)} & \text { Corrientes de fases } R, S \text { y T en función del tiempo } \\ n=1,3,5 & \text { Orden del armónico } \\ a_{n} & \text { Angulo de fase } \\ k & k=0,1,2 \\ I_{N} & \text { Valor eficaz de la corriente de neutro } \\ I_{f} & \text { Valor eficaz de la corriente total de fase } \\ m & \text { Relación porcentual de la carga en la fase T sobre la carga de la fase } R . \\ T H D_{U} T H D_{I} & \text { Índice de distorsión armónica total de tensión, corriente } \\ Z_{\text {eq, }}, X_{\text {eq }}, R_{\text {eq }} & \text { Impedancia, reactancia, resistencia equivalente de línea } \\ X_{N}, R_{N} & \text { Reactancia, resistencia del conductor de neutro } \\ U_{R}, U_{S}, U_{T} & \text { Valor eficaz de las tensiones de fases } R \text {, S y } T \\ U_{3, R} & \text { Valor eficaz del componente armónico de orden 3 de la tensión de fase } R \\ I_{1, N}, I_{3, N}, I_{9, N}, I_{15, N} I_{21, N} & \text { Valor eficaz de los componentes armónicos de corriente de neutro } \\ I_{1, f}, I_{3, f}, I_{9, f}, I_{15, f}, I_{21, f} & \text { Valor eficaz de los componentes armónicos de corriente de fase }\end{array}$

\section{REFERENCIAS}

Arthur R. y R.A. Shanahan; Neutral Currents in Three Phase Wye Systems, Actas del IEEE, 7th. International Conference on Harmonics and Quality of Power, Las Vegas, USA 16 al 18 de Octubre (1996).

Batrinu F. y otros seis autores; Experimental evaluation of unbalance and distortion indicators in three-phase Systems with Neutral, Actas del IEEE Power Tech, Lausanne, Suiza 1 al 5 de julio (2007).

Brugnoni M.; Efecto de las Cargas Distorsivas en las Redes de Distribución Eléctrica, Actas del Duodécimo Encuentro Regional Iberoamericano del CIGRÉ, 1-7, Foz Do Iguazú, Brasil, 20 al 24 de mayo (2007).

Cadavid D y L. Gallego; Armónicos y problemas de "power quality" en el conductor de neutro en sistemas trifásicos, Scientia Et Técnica: 22(9), 19-24 (2003).

Chan M., K. Lee y M. Fung; A case study of harmonic currents generated from a computer centre in a office building, Architectural Science Review: 50(3), 274-280 (2007).

Chicco G., P. Postolache y C. Toader; Analysis of three-phase systems with neutral under distorted and unbalanced conditions in the symetrical component-based framework, IEEE Transactions On Power Delivery: 22(1), 674-683 (2007).

Demoulias C, Z. Kampouri y K. Gouramanis; Natural canceling of current harmonics in office loads and its effect upon the transmission capacity of distribution cables, Actas del IEEE International Symposium on Industrial Electronics, Cambridge, Reino Unido 30 de junio al 2 de julio (2008).

Desmet J. y otros cuatro autores; Analysis of the neutral conductor current in a three phase supplied network with non-linear single phase loads, Actas del Electric Machines and Drives Conference, IEMDC IEEE International, Cambridge, USA 17 al 20 de junio (2001). 
Ghijselen J.A., W.R. Ryckaert, D.P. Zenner y J.A. Melkebeek; Considering Peak Rectifier Behaviour for Voltage Distortion Calculations in Power Distribution System, Industrial an Commercial Power Systems, 2003, IEEE Technical Conference, 5-11, 4 al 8 de mayo (2003).

Jou H. L., y otros cuatro autores; Analysis of zig-zag transformers applyng in the three-phase for wire distribution power system, IEEE Transactions On Power Delivery: 20(2), 1168-1173 (2005).

Larsson E., M. Wahlberg, M. Bollen y C. Lundmark; Waveform distortion at computer festivals; 2002 to 2008, Actas del $13^{\circ}$ International Conference on Harmonics and Quality of Power, Wollongong, Australia 28 de setiembre al 3 de octubre (2008).

Lowenstein M.; Eliminating harmonic neutral current problems, Actas del IEEE PES Transmission and Distribution Conference and Exposition, Bogotá, Colombia 13 al 15 de agosto (2008).

Moore P. e I. Portugués; The influence of personal computer processing modes on line current harmonics, IEEE Transactions on Power Delivery: 18(4), 1363-1368 (2003).

Paraiso, D., É. Ngandui, M. de Montigny y P. Siccard; Characterization of neutral and line current harmonics in three-phase computer power systems, Industrial Electronics Society, Actas del $31^{\circ}$ Conferencia Anual de la IEEE- IECON, Raleigh, USA 6 al 10 de noviembre (2005).

Rodriguez P. y otros cuatro autores; Cancellation of neutral current harmonics by using a four-branch star hybrid filter, Actas del IEEE Conference Power Electronics Specialist, Isla de Rodas, Grecia 15 al 19 de junio (2008).

Sainz L., J. Pedra, J. y J. Mesas; Study of neutral conductor current in three-phase networks with single-phase converters, IEEE Transactions on Power Delivery: 21(3), 1466-1476 (2006).

Song Q. y otros cinco autores; A novel approach to eliminate zero-sequence harmonics current, Actas del IEEE Asia Pacific Conference on Circuits and Systems, Macao, China 30 de noviembre al 3 de diciembre (2008).

Suárez J; G. di Mauro, D. Anaut y C. Agüero; Cancelación Parcial de Corrientes Armónicas Provocadas por Cargas Residenciales No Lineales, Información Tecnológica: 16(3), 63-70 (2005). 